Pacific Journal of Mathematics

GENERALIZED INDUCTIVE LIMIT TOPOLOGIES AND 


\title{
GENERALIZED INDUCTIVE LIMIT TOPOLOGIES AND BARRELLEDNESS PROPERTIES
}

\author{
WOLFGANG RUESS
}

\begin{abstract}
For a locally convex space $(X, \tau)$ and an increasing sequence $\left(A_{\nu}\right)_{\nu \in N}$ of convex, circled subsets of $X$ the generalized inductive limit topology related to $(X, \tau)$ and $\left(A_{\nu}\right)_{\nu \in N}$ is defined to be the finest locally convex topology on $X$ agreeing with $\tau$ on the sets $A_{\nu}, \nu \in N$. Several results on the classification and the inheritance properties of various types of barrelledness and their evaluable analogs are shown to be consequences only of a few basic properties of such an inductive limit topology and, in this way, are deduced and extended in a unified manner.
\end{abstract}

Introduction. In recent years several types of barrelledness and their respective inheritance properties have been considered, cf. [4-7], [14], [19-21] and [23-30]. The methods of the proofs of these various results are rather different. It is the primary object of this paper to develop a unified approach to a great part of the quoted results and, simultaneously, to present some natural extensions. This is essentially done by showing that, after all, these results are "generalized inductive limit results" in the sense of Garling [9].

The central notions are that of an absorbent sequence $\mathscr{A}=\left(A_{\nu}\right)_{\nu \in N}$ of subsets $A_{\nu}$ of a locally convex space $(X, \tau)$ and the associated generalized inductive limit topology $\eta_{\varkappa}$ :

Definition. Let $(X, \tau)$ be a locally convex space with topology $\tau$.

(1) An increasing sequence $\left(A_{\nu}\right)_{\nu \in N}$ of convex, circled subsets of $X$ is called absorbent (resp. bornivorous) if every $x \in X$ (resp. every bounded subset of $X$ ) is absorbed by $A_{\nu}$ for some $\nu \in N$ (see [5]).

(2) If $\mathscr{A}=\left(A_{\nu}\right)_{\nu \in N}$ is any increasing sequence of convex, circled subsets of $X$ then we denote by $\eta_{\diamond}$ the finest locally convex topology on $X$ agreeing with $\tau$ on the sets $A_{\nu}$ for every $\nu \in N$. (In case $\mathscr{A}=\left(A_{\nu}\right)_{\nu \in N}$ is an absorbent sequence in $X$ the topology $\eta_{\mathscr{A}}$ has been investigated in great detail by Garling [9] and Roelcke [16].)

We shall be concerned with the following classes of locally convex spaces: A locally convex space $(X, \tau)$ is said to be of type

(1) Baire-like ((1b) b-Baire-like) if it is not the union of an absorbent (bornivorous) sequence of nowhere dense subsets of $X$;

(2) quasi-Baire ((2b) b-quasi-Baire) if it is barrelled (evaluable) 
and not the union of an absorbent (bornivorous) sequence of nowhere dense linear subspaces of $X$;

( 3 ) barrelled $((3 \mathrm{~b})$ evaluable) if every (bornivorous) barrel is a neighbourhood of zero in $X$;

(4) $\boldsymbol{\aleph}_{0}$-barrelled ((4b) $\aleph_{0}$-evaluable) if every (bornivorous) barrel which is the countable intersection of convex, circled, closed neighbourhoods of zero, is itself a neighbourhood of zero in $X$;

$\left(4^{*}\right) \quad(\mathrm{L})\left(\left(4 \mathrm{~b}^{*}\right)(\mathrm{Lb})\right)$ if for every absorbent (bornivorous) sequence $\mathscr{A}=\left(A_{\nu}\right)_{\nu \in N}$ in $X$ we have $\eta_{\mathscr{N}}=\tau$;

(5) $\sigma$-barrelled $((5 b) \sigma$-evaluable $)$ if every $\sigma\left(X^{\prime}, X\right)-\left(\beta\left(X^{\prime}, X\right)-\right)$ bounded sequence in $X^{\prime}$ is equicontinuous;

(6) (C) $((6 \mathrm{~b})(\mathrm{Cb}))$ if every $\sigma\left(X^{\prime}, X\right)-\left(\beta\left(X^{\prime}, X\right)-\right)$ bounded subset of $X^{\prime}$ is relatively- $\sigma\left(X^{\prime}, X\right)$-countably compact;

(7) (S) ((7b) (Sb)) if every $\left(\beta\left(X^{\prime}, X\right)\right.$-bounded) $\sigma\left(X^{\prime}, X\right)$-Cauchysequence in $X^{\prime}$ is $\sigma\left(X^{\prime}, X\right)$-convergent;

(8) (LC) ((8b) (LCb)) if for every absorbent (bornivorous) sequence $\mathscr{A}=\left(A_{\nu}\right)_{\nu \in N}$ in $X$ we have $\eta_{,} \subset \tau\left(X, X^{\prime}\right)$, where $\tau\left(X, X^{\prime}\right)$ is the Mackey topology on $X$;

(9) (B) if for every absorbent sequence $\mathscr{A}=\left(A_{\nu}\right)_{\nu \in N}$ in $X$ we have $\eta_{\star} \subset \tau_{b}$, where $\tau_{b}$ is the associated bornological topology for $(X, \tau)$.

For the classes $(n), n \in\{1, \cdots, 7\}$, see [19]; the classes $\left(4^{*}\right),\left(4 b^{*}\right)$, (8) and (8b) have been introduced in [18]; for the class (5b) cf. [5]; the new classes (nb), $n \in\{1,2,6,7\}$, are the "evaluable analogs" of the respective spaces of type $(\mathrm{n}), n \in\{1,2,6,7\}$. Together with the classes (8) and (9) they are the natural supplement of Saxon's classification [19] of locally convex spaces: results about the properties (n) turn out to be consequences only of results about the respective properties (nb), $n \in\{1, \cdots, 8\}$ and property (B). This leads to natural and simplified proofs of classification- and inheritance-results related to Saxon's scheme of barrelledness properties.

Section 1 contains some basic properties of our classification scheme. In $\S \S 2$ and 3 we develop the technique which enables us to deduce the countable-codimensional inheritance properties of the types of barrelledness given above in a unified manner as "generalized inductive limit results". Section 4 contains a new characterization of barrelled (resp. evaluable) spaces by a Banach-Steinhaus property related to absorbent (resp. bornivorous) sequences. In $\S 5$ there are given various general examples of classes of spaces of the above scheme.

As for the notation we generally follow Horvath's text [12]. The notion "absorbent sequence" (resp. "bornivorous sequence") is abbreviated by "(as)" (resp. "(bs)"). Throughout this paper linear topological spaces are assumed to be hausdorff. The notion "locally 
convex linear topological space" is abbreviated by "l.c.s.".

A convex and circled subset of a linear space is called a disk. A bounded disk $B$ in an l.c.s. $(X, \tau)$ is called completing, if $\left(X_{B}, q_{B}\right)$ is a Banach space, where $\left(X_{B}, q_{B}\right)$ is the linear span of $B$ in $X$ endowed with the norm $q_{B}, q_{B}$ being the Minkowski functional of $B$ with respect to $X_{B}$. An l.c.s. $(X, \tau)$ is called Mackey-complete if every bounded and closed disk in $(X, \tau)$ is completing.

If $(X, \tau)$ is an l.c.s. then we denote by $\mathscr{C}=$ the filter of $\tau$ neighbourhoods of zero. A disk $U$ in $(X, \tau)$ is called $\boldsymbol{\aleph}_{0}$-barrel if it is absorbing in $X$ and if there exists a sequence $\left(U_{2}\right)_{\nu \in N}$ of closed disks $U_{\nu} \in \mathscr{Z}_{\mathrm{C}}$. such that $U=\bigcap_{\nu \in N} U_{\nu}$.

1. Classification results. Let $(X, \tau)$ be an l.c.s. and $\mathscr{A}=\left(A_{\nu}\right)_{\nu \in N}$ an (as) in $X$. The following results about $\eta$. will be needed (cf. [9], [16]):

(1a) A $\tau$-bounded subset of $X$ is $\eta_{\mathscr{}}$-bounded if and only if it is absorbed by some $\bar{A}_{\nu}$; (1b) $\eta_{\nu}$, does not change if any $A_{\nu}$ is replaced

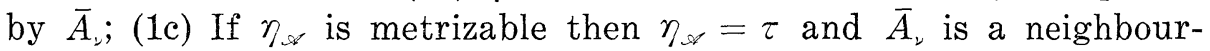
hood of zero for sufficiently large $\nu$; (1d) $\eta_{*}$ has a neighbourhoodbase of zero consisting of $\boldsymbol{\aleph}_{0}$-barrels in $(X, \tau)$; if, in addition, $\left(\bar{A}_{\nu}\right)_{\nu \in N}$ is a (bs) in $(X, \tau)$, then $\eta_{\nsim}$ has a neighbourhood-base of zero consisting of bornivorous $\boldsymbol{N}_{0}$-barrels in $(X, \tau)$; (1e) If for any $\lambda, \mu \in N$ there exists $\nu \in N$ such that $A_{\lambda}+A_{\mu} \subset A_{\nu}$, then an absorbing disk $V$ in $X$ is an $\eta_{\infty}$-neighbourhood of zero as soon as $V \cap A_{n}$ is a $\tau \mid A_{n}$-neigbourhood of zero for every $n \in N$.

Lemma 1.1. Let $(X, \tau)$ be an l.c.s. and let $\left(A_{\nu}\right)_{\nu_{\in N}}$ be an (as) in $X$ consisting of closed sets. Then every bounded and completing disk $B$ of $X$ is absorbed by some $A_{\nu}$.

This follows from the fact that $\left(X_{B}, q_{B}\right)$ is a Banach space, $\left(A_{\nu} \cap X_{B}\right)_{\nu \in N}$ is an (as) of closed sets in $\left(X_{B}, q_{B}\right)$ and hence one of the $A_{\nu} \cap X_{B}$ 's is a neighbourhood of zero in $\left(X_{B}, q_{B}\right)$. This argument and Examples 1.1 and Corollary 1.5 below show, that, as far as $B$ is concerned, it suffices to assume that $\left(X_{B}, q_{B}\right)$ be barrelled.

Lemma 1.2. Let $(X, \tau)$ be an l.c.s.

(1) $(X, \tau)$ has property (B) if and only if every (as) of closed sets in $X$ is a (bs) in $X$. In particular, every l.c.s. $(X, \tau)$ such that $\left(X_{B}, q_{B}\right)$ is barrelled for every closed, bounded disk $B$ in $X$ has property (B).

(2) If $(X, \tau)$ has property (Sb) then it has property (LCb).

((1) extends Cor. 1 in [5], Cor. 2.4 in [19] and Thm. 6 in [25], 
see Lemma 6.3 in [18].)

Proofs. (1) is a consequence of results (1a) and (1b) and of the remarks following Lemma 1.1. (2): Assume that $(X, \tau)$ has (Sb) and let $\mathscr{A}=\left(A_{\nu}\right)_{\nu \in N}$ be a $(\mathrm{bs})$ in $X$. If we denote by $\mathrm{cpl}{ }_{\mathscr{N}} X^{\prime}$ the completion of $X^{\prime}=(X, \tau)^{\prime}$ with respect to the topology of uniform con-

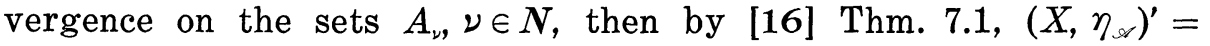
cpl $X_{\mathscr{}}^{\prime}$. But by assumption and by the general result [13], $\S 18,4(4)$ on the inheritance of the convergence of Cauchy filters we have cpl $X_{\mathscr{A}} X^{\prime}=X^{\prime}$.

Taking into account the results of [18] and [19] it is now clear that every space of type (n) (resp. (nb)) is of type $(n+1), n \in$ $\{1, \cdots, 8\}$ (resp. $((n+1) b), n \in\{1, \cdots, 7\})$. Moreover, every space of type (4) (resp. (4b)) is of type $\left(4^{*}\right)$ (resp. $\left(4 b^{*}\right)$ ). None of the converse inclusions holds. This is shown by the examples in $\S 5$.

Lemma 1.2 yields:

COROLlary 1.3. Let $(X, \tau)$ be an l.c.s. for which there exists a sequence $\left(B_{\nu}\right)_{\nu \in N}$ of bounded subsets such that $\bigcup_{\nu \in N} B_{\nu}$ is absorbing in $X$. If $(X, \tau)$ has property $(\mathrm{B})$, then $(X, \tau)$ has a fundamental sequence of bounded subsets.

For the case of a barrelled space this has been proved by Valdivia (see Corollary. 2.6 of [25]).

Clearly every space of type (n) is of type (nb), $n \in\{1, \cdots, 8\}$. Examples 1.2 below show that none of the converse inclusions is valid. But we do get the following general result which shows the special character of property (B):

Lemma 1.4. If $(X, \tau)$ is an l.c.s. of type (nb) (resp. of type $\left(4 \mathrm{~b}^{*}\right)$ ) and has property (B), then it is of type (n) (resp. of type $\left(4^{*}\right)$ ), $n \in\{1, \cdots, 8\}$.

This follows from result (1b) and Lemma 1.2 (1).

The following examples, in a certain sense, justify the introduction of $b$-Baire-like spaces:

EXAMPLeS 1.1. Every metrizable l.c.s. is b-Baire-like.

Proof. If $(X, \tau)$ is metrizable and $\mathscr{A}=\left(A_{\nu}\right)_{\nu \in N}$ a $(\mathrm{bs})$ in $X$, then $\eta_{\star}=\tau$, for $(X, \tau)$ has property (Lb) ([18] Examples 6.2, (3)). In particular, $\eta_{\star}$ is metrizable. Hence, by result (1c), $\bar{A}_{\nu}$ is a $\tau$ - 
neighbourhood of zero for some $\nu \in N$.

In [19] Saxon proved the following proposition: A metrizable l.c.s. is Baire-like if and only if it has property (S) (Theorem 27 of [19]). As an easy consequence only of Lemma 1.4 there can be given the following extension of this result:

Corollary 1.5. If $(X, \tau)$ is a b-Baire-like l.c.s. then $(X, \tau)$ is Baire-like if and only if it has property (B).

By Examples 1.1 and Examples 5.6 and 5.7 of $\S 5$ this corollary also extends Proposition 6.8 of [18]. Moreover, the concepts " $b$-Bairelike" and property " $B$ " seem to be the natural setting of this result.

Another property of metrizable l.c.s. is also a consequence of the fact that these spaces are $b$-Baire-like:

Proposition 1.6. Any b-Baire-like (DF)-space is normable.

(It should be noted here that an evaluable (DF)-space is in general not bornological.)

Corollary 1.5 yields the following Examples 1.2: Every nonbarrelled metrizable l.c.s. is of type (nb) for all $n \in\{1, \cdots, 8\}$, but not of type (n) for any $n \in\{1, \cdots, 9\}$.

2. Basic results on generalized inductive-limit topologies. The first lemma will yield one of the main tools for the techniques of this paper (Theorem 2.4 below). On the other hand, it also contains fundamental results of the papers [5] ([5], Theorem, 2) and [25] ([25], Lemma 1) as special cases.

Lemma 2.1. Let $X$ be a linear space and $\rho_{1}$ and $\rho_{2}$ locally convex linear topologies on $X$ such that $\rho_{1} \subset \rho_{2}$. Let $\left(B_{\nu}\right)_{\nu \in N}$ be an (as) in $X$ and let $\left(\alpha_{\nu}\right)_{\nu \in N} \subset \boldsymbol{R}^{+}$such that $\alpha_{\nu} \leqq \alpha_{\nu+1}, \nu \in N$. If for any sequence $\left(U_{\nu}\right)_{\nu \in N}$ of disks $U_{\nu} \in \mathscr{U}_{\rho_{1}}$ the set $U=\bigcap_{\nu \in N}\left(U_{\nu}+\alpha_{\nu} B_{\nu}\right)$ is a $\rho_{2}$-neighbourhood of zero, then we have:

If $\mathscr{F}$ is any $\rho_{2}$-Cauchy filterbase on $\bigcup_{\nu \in N} B_{\nu}$ then the filterbase $\mathscr{F}+\mathscr{U}_{\rho_{1}}=\left\{F+U \mid F \in \mathscr{F}, U \in \mathscr{U}_{\rho_{1}}\right\}$ induces a $\rho_{1}$-Cauchy filterbase on $\left(1+\alpha_{n}\right) B_{n}$ for some $n \in N$.

Corollary 2.2. Let $(X, \tau)$ be an 1.c.s., let $A$ be a linear subspace of $X$ and $\mathscr{A}=\left(A_{\nu}\right)_{\nu \in N}$ an (as) in $A$. Assume that $A$ has property $\left(H_{\mathscr{A}}\right)$ : Every sequence $\left(a_{\nu}^{\prime}\right)_{\nu \in N}$ in $A^{\prime}$ with the property that for every $n \in N$ there exists $\nu(n) \in N$ such that $\left(\alpha_{\mu}^{\prime}\right)_{\mu \geqq \nu(n)} \subset A_{n}^{0}$ is equicontinuous. Then: 


$$
\widehat{\bigcup}_{\nu \in N} A_{\nu}=\bigcap_{\varepsilon>0}(1+\varepsilon) \bigcup_{\nu \in N} \bar{A}_{\nu} .
$$

In particular, the conclusion holds if $A$ is $\sigma$-barrelled ( $\sigma$-evaluable) and $\left(A_{\nu}\right)_{\nu \in N}$ is any (as) ((bs)) in $A$, or if $A$ is sequentially-barrelled in the sense of [28], $\left(B_{\nu}\right)_{\nu \in N}$ is an (as) in $A$ and $A_{\nu}=\nu B_{\nu}$.

This is essentially Theorem 2.1 of [6] and Theorem 2 in [5].

CoROLlaRY 2.3. Let $\mathscr{A}=\left(A_{\nu}\right)_{\nu \in N}$ be an increasing sequence of disks in an l.c.s. $(X, \tau)$. Then we have:

If $\mathscr{F}$ is any $\eta_{\mathscr{N}}$-Cauchy filterbase on $\bigcup_{\nu \in N} A_{\nu}$, then the filterbase $\mathscr{F}+\mathscr{U}_{\eta_{\Im}}$ induces an $\eta_{\mathscr{N}}$-Cauchy filterbase on $n A_{n}$ for some $n \in N$.

THEOREM 2.4. Let $\mathscr{A}=\left(A_{\nu}\right)_{\nu \in N}$ be an increasing sequence of disks in an l.c.s. $(X, \tau)$ and let $A=\operatorname{span}\left(\bigcup_{\nu \in N} A_{\nu}\right)$. Then we have:

(1) $\bar{A}^{\eta \otimes}=\mathrm{U}_{\nu \in N} \nu \bar{A}_{\nu}^{\eta ⿻}$

(2) If, in addition, every $A_{\nu}$ is closed, then $A$ is $\eta_{\rtimes}$-closed and every (algebraic) complement $B$ of $A$ is a topological complement with respect to $\eta_{-}$and $\eta_{*} \mid B=\rho_{B}$, where $\rho_{B}$ denotes the finest locally convex topology on $B$.

REMARKs 1. Lemma 1 of [25] is a special case of Lemma 2.1. Direct consequences of Corollary 2.2 are the following two results (Corollary $2 \mathrm{~b}$ and Corollary $2 \mathrm{c}$ of [5]):

(a) In a $\sigma$-barrelled ( $\sigma$-evaluable) l.c.s. the union of an (as) $((\mathrm{bs}))$ of closed sets contains a barrel.

(b) $A \sigma$-barrelled ( $\sigma$-evaluable) 1.c.s. is complete whenever it contains an (as) ((bs)) of complete sets.

These results will be needed in $\S \S 4$ and 5 .

Let us note here that result (b) (and thus Corollary 2.2) is false if $X$ is no longer supposed to be $\sigma$-barrelled but only to be of type (C): Examples 5.4 in $\S 5$ show that the weak dual of an infinitedimensional (F)-space has property (C). But such a space has a (bs) of compact sets without being complete.

2. Theorem 2.4 is an easy consequence of Corollary 2.3. This corollary, moreover, also yields the following result: If $\mathscr{A}=\left(A_{\nu}\right)_{\nu \in N}$ is an (as) in an l.c.s. $(X, \tau)$, then $\left(X, \eta_{\infty}\right)$ is complete if and only if $\bar{A}_{\nu}$ is $\tau$-complete for every $\nu \in N$. Thus the conclusion of proposition (1b) above also holds true in case $X$ has property (L) ((Lb)). This in particular yields the well known result that the strict inductive limit of an increasing sequence of complete l.c.s. is complete.

Proof of Lemma 2.1. (The proof is a modification of the one given 
for Theorem 1 in [15].) Assume on the contrary that there exist sequences $\left(F_{\nu}\right)_{\nu \in N}, F_{\nu} \in \mathscr{F}$, and $\left(U_{\nu}\right)_{\nu \in N}, U_{\nu} \in \mathscr{U}_{\rho_{1}}$ and $U_{\nu}$ a disk, such that $\left(F_{\nu}+U_{\nu}\right) \cap\left(1+\alpha_{\nu}\right) B_{\nu}=\varnothing$. Since the set $U=\bigcap_{\nu \in N}\left(U_{\nu}+\alpha_{\nu} B_{\nu}\right)$ is a $\rho_{2}$-neighbourhood of zero, there exists $F \in \mathscr{F}$ such that $F-F \subset U$. Let $x \in F$; then there exists $n \in N$ such that $x \in B_{n}$. Hence $F \subset x+$ $U \subset B_{n}+U_{n}+\alpha_{n} B_{n}=\left(1+\alpha_{n}\right) B_{n}+U_{n}$ and thus $F \cap F_{n}=\varnothing$, which is a contradiction.

Proof of Corollary 2.2. Let $\varepsilon>0$ and $x \in \overline{\mathrm{U}_{\nu \in N} A_{\nu}}$. In the notation of Lemma 2.1 let $X=A, \rho_{1}=\sigma\left(A, A^{\prime}\right), \rho_{2}=\tau \mid A, B_{\nu}=A_{\nu}, \alpha_{\nu}=\varepsilon$ and $\mathscr{F}=\left(x+\mathscr{U}_{\tau}\right) \cap \bigcup_{\nu \in N} A_{\nu}$. Then Lemma 2.1 completes the proof for the case $\sigma$-barrelled ( $\sigma$-evaluable). For a proof of the general case let $U=\bigcap_{\mu_{N} N}\left(F_{\mu}^{0}+\varepsilon A_{\mu}\right)$, where $F_{\mu}$ is a finite subset of $A^{\prime}$. Since $B_{\mu}=\left(F_{\mu}^{0}+\varepsilon A_{\mu}\right)^{0}$ is a compact absorbing disk in a finite-dimensional space, there exists a finite subset $E_{\mu} \subset 2 B_{\mu} \subset 2\left(\left(\Gamma F_{\mu}\right) \cap\left(\varepsilon A_{\mu}\right)^{0}\right)$ such that $B_{\mu} \subset \Gamma E_{\mu}$, and thus $\left(2 \bigcup_{\mu_{\in N}} E_{\mu}\right)^{0} \subset U . \quad B y\left(H_{\varkappa}\right)$, the set $\bigcup_{\mu \in N} E_{\mu}$ is equicontinuous.

Proof of Corollary 2.3. Let $A=\operatorname{span}\left(\bigcup_{\nu \in N} A_{\nu}\right)$. In the notation of Lemma 2.1 let $X=A, \rho_{1}=\rho_{2}=\eta_{s} \mid A, B_{\nu}=\nu A_{\nu}, \alpha_{\nu}=1$.

The next theorem contains the key result for the consideration of the evaluable classes of our classification scheme:

Notation. Let $(X, \tau)$ be an l.c.s. and let $\mathscr{B}$ be any family of bounded disks in $X$. Then $\tau_{\mathscr{A}}$ denotes the finest locally convex topology on $X$ such that the canonical embeddings $\kappa_{B}:\left(X_{B}, q_{B}\right) \rightarrow$ $X, B \in \mathscr{B}$, are continuous.

$\tau_{\mathscr{O}}$ is the finest locally convex topology on $X$ for which the sets $B \in \mathscr{B}$ are bounded.

Theorem 2.5. Let $(X, \tau)$ be an l.c.s. and let $A$ be a finitecodimensional linear subspace of $X, X=A \oplus \operatorname{span}\left\{x_{1}, \cdots, x_{n}\right\}$ (algebraically) for some linear independent set $\left\{x_{1}, \cdots, x_{n}\right\} \subset X$. Let $\mathscr{B}$ be any family of bounded disks in $X$. Then we have: If $\left(A_{\nu}\right)_{\nu \in N}$ is an (as) in $A$ such that every $B \cap A, B \in \mathscr{B}$, is absorbed by some $A_{\nu}$, then every $B \in \mathscr{B}$ is absorbed by $\left(\bar{A}_{\nu}+\Gamma\left\{x_{1}, \cdots, x_{n}\right\}\right)$ for some $\nu \in N$.

Proof. It suffices to carry out a proof for the case codim $A=1$. Let $X=A \oplus \operatorname{span}\{x\}$ for some $x \in X \backslash A$. We define:

$\eta=$ the finest locally convex topology on $X$ such that

$$
\eta\left|\left(A_{\nu}+\Gamma\{x\}\right)=\tau\right|\left(A_{\nu}+\Gamma\{x\}\right) \text { for every } \nu \in N .
$$


$\eta_{A}=$ the finest locally convex topology on $A$ such that $\eta_{A}\left|A_{\nu}=\tau\right| A_{\nu}$ for every $\nu \in N$.

By assumption and result (1a) we have: $\eta_{A} \subset(\tau \mid A)_{\mathscr{B} \cap A}$. Clearly we have: $\eta \mid A \subset \eta_{A}$. Corollary 1.1 of [26] yields: $(\tau \mid A)_{\mathscr{B} \cap A}=\tau_{\mathscr{B}} \mid A$. Hence we know: $\eta\left|A \subset \tau_{\mathscr{B}}\right| A$. We now show: $(*) \eta \subset \tau_{\mathscr{B}}$.

Let $U$ be a convex, circled $\eta$-neighbourhood of zero in $X$, which, by result (1d), can be assumed to be $\tau$-closed. We know already that $U \cap A$ is a $\tau_{B} \mid A$-neighbourhood of zero.

Case 1. $A$ is $\tau_{\mathscr{B}}$-dense in $X$. Then, since $U=\bar{U}_{\mathscr{C}} \supset \bar{U}_{\mathscr{B}} \supset \overline{U \cap A}_{\mathscr{B}}$, $U$ is a $\tau_{\mathscr{B}}$-neighbourhood of zero.

Case 2. $A$ is $\tau_{\mathscr{B}}$-closed. Then $U \cap A+\Gamma\{x\}$ is a $\tau_{\mathscr{B}}$-neighbourhood of zero. But $U$ absorbs $U \cap A+\Gamma\{x\}$, hence $U$ itself is a $\tau_{\mathscr{B}}$-neighbourhood of zero. Thus we have shown $(*)$. This means that the sets $B \in \mathscr{B}$ are $\eta$-bounded. Thus, by result (1a), every $B \in \mathscr{B}$ is absorbed by some $\left(\bar{A}_{\nu}+\Gamma\{x\}\right)$.

Finally, the following result will be needed later on:

Lemma 2.6. Let $(X, \tau)$ be a hausdorff linear topological space and let $\eta$ be a linear topology on $X$ which is finer than $\tau$. Then we have: If $A$ is a r-closed subset of $X$ such that $\eta|A=\tau| A$ and $B$ is an $\eta$-compact subset of $X$, then $\eta|(A+B)=\tau|(A+B)$.

(This generalizes the fact that, under the assumptions of the lemma, $\eta$ and $\tau$ coincide on $\eta$-compact subsets of $X$.)

The proof of Lemma 2.6 is a consequence of the following observation: Let $(X, \tau)$ be a hausdorff topological space and let $\left(x_{r}\right)_{r \in \Gamma}$ be a net in $X$. If there is an $x \in X$ which is an accumulation point of every subnet of $\left(x_{\gamma}\right)_{\gamma \in \Gamma}$, then $\left(x_{\gamma}\right)_{\gamma \in \Gamma}$ converges to $x$. If, given the situation described in Lemma 2.6, $\left(x_{r}\right)_{r \in \Gamma}$ is a net in $A+B$ which is $\tau$-convergent to $x \in A+B$, then, by the $\eta$-compactness of $B$, the argument above applies to $\eta$ and $x$.

3. Inheritance properties. In this section we are concerned with the inheritance of properties (n), $n \in\{1, \cdots, 9\}$, and (nb), $n \in$ $\{1, \cdots, 8\}$, by linear subspaces of countable codimension.

First let us list some other permanence properties which will be needed later on:

Properties (n), (nb), $n \in\{3,4,5,7,8\},\left(4^{*}\right)$ and $\left(4 b^{*}\right)$ are inherited by locally convex final topologies, hence by inductive limits, direct sums, quotients and products. (As for the conclusion for products 
see [3].)

Properties (n) and (nb) for $n \in\{1,2\}$ are, in general, not inherited even by countable inductive limits: Examples 5.2 of $\S 5$ include a (Ptak, nuclear) regular (LB)-space which is not b-quasi-Baire.

As for products, however, there is the following positive result: Every product of b-Baire-like resp. b-quasi-Baire 1.c.s. is b-Bairelike resp. b-quasi-Baire.

(This is a consequence of Theorem 2.9 of [19] and the following two results.)

If $(X, \tau)$ is a $\sigma$-barrelled ( $\sigma$-evaluable) l.c.s. such that its completion is Baire-like resp. quasi-Baire, then $(X, \tau)$ is Baire-like resp. quasi-Baire ( $b$-Baire-like resp. $b$-quasi-Baire). (This is a variant of Corollary 2.d. of [5].)

If a dense linear subspace $A$ of an l.c.s. $(X, \tau)$ has any of the properties (n), (nb), $n \in\{1, \cdots, 5\}$, then so does $X$. In particular, the completion of an l.c.s. of type (nb) is of type (n), $n \in\{1, \cdots, 5\}$ (see Lemma 1.4).

In general, none of the properties of our classification scheme is inherited by (closed) linear subspaces. Yet there can be obtained positive results in the countable-codimensional case. These will now be derived in a unifield manner as easy consequences of the results in $\S 2$.

Notation. A linear subspace $A$ of an l.c.s. $(X, \tau)$ is said to have property (b) if for every bounded subset $B$ of $(X, \tau)$ the codimension of $A$ in $\operatorname{span}\{A \cup B\}$ is finite (see [23], [30]).

If $(X, \tau)$ is an l.c.s. of type $(B)$, then every closed linear subspace of $X$ of countable codimension has property (b). This follows from Lemma 1.2 (1). For further comments on property (b) cf. [23], [29] and $[30]$.

TheOREM 3.1. Let $(X, \tau)$ be an l.c.s. and let $A$ be a linear subspace of $X$ of countable codimension.

(1) If $X$ is of type (n), $n \in\{1, \cdots, 9\}$, or $\{\mathrm{L}\}$, then $A$ is of the same type.

(2) If $A$ has property (b) and $X$ is of type (nb), $n \in\{1, \cdots, 8\}$, or (Lb), then $A$ is of the same type.

(3) If $X$ is of type (nb) (resp. (Lb)) and has property (B), then $A$ is of type (n) (resp. (L)), $n \in\{1, \cdots 8\}$.

Propositions (1) and (2) unify and extend the corresponding results in [4], [7], [14], [19-20], [23-25], ]29-30]. The cases (n), $n \in\{8,9\},\left(4^{*}\right),(\mathrm{nb}), n \in\{1,2,5,6,7,8\}$, and $\left(4 \mathrm{~b}^{*}\right)$ are new.

Proposition (3) is a consequence of (1) and Lemma 1.4 . 
Theorem 4 of [23] is a special case of (3) for $n=4$.

Since we want to prove somewhat more general results, the proof of Theorem 3.1 will be carried out in two steps.

Case 1. A is closed.

Proposition 3.2. Let $(X, \tau)$ be an l.c.s. which is $\sigma$-barrelled ( $\sigma$-evaluable) or has property $(\mathrm{L})((\mathrm{Lb}))$. Let $A$ be a closed linear subspace of $X$ (with property (b)) of countable codimension. Then every complement $B$ of $A$ is a topological complement and carries the finest locally convex topology.

For a $\sigma$-barrelled 1.c.s. this is Theorem 1.1 of [19], Theorem 6 of [7] for a barrelled l.c.s. and Proposition 2 of [20] for Mackey spaces with property (S).

Proposition 3.3. Let $(X, \tau)$ be an l.c.s. of type (LC) $((\mathrm{LCb}))$ and let $A$ be a closed linear subspace of $X$ (with property (b)) of countable codimension. Let $B$ be any complement of $A$ and let $\eta$ be the finest locally convex topology on $X$ such that $\eta|A=\tau| A$.

(1) $\eta$ is compatible with the dual pair $\left(X, X^{\prime}\right)$ and $(X, \eta)=$ $A \oplus B$ is a topological decomposition with respect to $\eta$ and $\eta \mid B=\rho_{B}$, where $\rho_{B}$ denotes the finest locally convex topology on $B$.

(2) Any extension of a continuous seminorm on $A$ to a seminorm on $X$ is $\tau\left(X, X^{\prime}\right)$-continuous.

(3) Any extension of a continuous linear functional on $A$ to a linear functional on $X$ is continuous.

(For the special case of a space of type (S) (3) is Lemma 2 of [20].)

Proof of Proposition 3.2. We prove the case $\operatorname{codim} A=\boldsymbol{\aleph}_{0}$. Let $\left(x_{\nu}\right)_{\nu \in N}$ be a Hamel base for $B$. Then the sequence $(A+$ $\left.\Gamma\left\{x_{1} \cdots, x_{\nu}\right\}\right)_{\nu \in N}$ is an (as) in $X$. If $A$ has property (b), then, by Theorem 2.5 , it is also a (bs) in $X$. Case (L) $((\mathrm{Lb}))$ : Let $\eta$ be the finest locally convex topology on $X$ agreeing with $\tau$ on $A$. Then, by Lemma 2.6, we have: $\eta\left|\left(A+\Gamma\left\{x_{1}, \cdots, x_{\nu}\right\}\right)=\tau\right|\left(A+\Gamma\left\{x_{1}, \cdots, x_{\nu}\right\}\right)$ for every $\nu \in N$. Hence $\eta=\tau$ and Theorem 2.4 (2) completes the proof for this case. Case $\sigma$-barrelled ( $\sigma$-evaluable): In the foregoing proof we replace $\tau$ by $\sigma\left(X, X^{\prime}\right)$. Then, again $\eta \mid\left(A+\Gamma\left\{x_{1}, \cdots, x_{\nu}\right\}\right)=$ $\sigma\left(X, X^{\prime}\right) \mid\left(A+\Gamma\left\{x_{1}, \cdots, x_{\nu}\right\}\right)$ for every $\nu \in N$. By result (1d) $\eta$ has a neighbourhood base of zero consisting of (bornivorous) $\boldsymbol{\aleph}_{0}$-barrels in $\left(X, \sigma\left(X, X^{\prime}\right)\right)$. Hence, by assumption, $\sigma\left(X, X^{\prime}\right) \subset \eta \subset \tau$. Again, Theorem 2.4 (2) completes the proof. 
Proof of Proposition 3.3. We prove (1), the other assertions follow easily. As in the proof of Proposition 3.2, case $(\mathrm{L})((\mathrm{Lb}))$, we get $\eta \subset \tau\left(X, X^{\prime}\right)$. Theorem 2.4 (2) completes the proof.

Proof of Theorem 3.1. By the above remark on property (b) and by Lemma 1.4 we need only prove (1), case (B), and (2). (B) has a straightforward proof. The cases (nb), $n \in\{1, \cdots, 5\}$, and $\left(4 b^{*}\right)$ are consequences of Proposition 3.2 and the results quoted at the beginning of this section. The cases $(\mathrm{nb}), n \in\{6,7\}$, are proved in a way similar to Proposition 3 in [14] by taking into account Proposition $3.3(3)$. Case (LCb): This property does not change if $\tau$ is replaced by any other locally convex topology on $X$ which is compatible with the dual pair $\left(X, X^{\prime}\right)$. Hence, with the notations and definitions of Proposition 3.3, $(X, \eta)$ has property (LCb) and thus $(A, \eta \mid A)=X / B$ has this property. But $\eta \mid A$ is compatible with the dual pair $\left(A, A^{\prime}\right)$.

Case 2. $A$ is dense in $X$ :

Lemma 3.4. Let $(X, \tau)$ be an l.c.s. and $A$ a dense linear subspace of $X$ such that for every (as) (resp. (bs)) $\left(A_{\nu}\right)_{\nu \in \mathbf{v}}$ in $A\left(\bar{A}_{\nu}\right)_{\nu \in Y}$ is an (as) (resp. a ((bs)) in X. Then we have:

(1) If $(X, \tau)$ is of type (n), $n \in\{1, \cdots, 9\} \backslash\{7\}$, or (L) (resp. of type (nb), $n \in\{1, \cdots, 8\} \backslash\{7\}$, or (Lb)), then $A$ is of the same type.

(2) If, in addition, codim $\mathrm{A} \leqq \boldsymbol{\aleph}_{0}$, then $A$ is of type (S) (resp. $(\mathrm{Sb}))$, whenever $X$ is of this type.

Theorem 3.1 now follows from the following proposition:

Proposition 3.5. Let $(X, \tau)$ be an l.c.s. and $A$ a linear subspace of $X$ of countable codimension.

(1) If $(X, \tau)$ is of type (LC) and $\left(A_{\nu}\right)_{\nu \in N}$ is an (as) in $A$, then $\left(\bar{A}_{\nu}\right)_{\nu \in N}$ is an (as) in $\bar{A}$.

(2) If $(X, \tau)$ is of type (LCb), if $A$ has property (b) and if $\left(A_{\nu}\right)_{\nu \in N}$ is a (bs) in $A$, then $\left(\bar{A}_{\nu}\right)_{\nu \in N}$ is a (bs) in $\bar{A}$.

Proposition 3.5 (1) yields the following extension of Lemma 3 of [20] and Theorem 5 of [29]:

Let $(X, \tau)$ be an 1.c.s. with property $(\mathrm{LC})$ and let $\left(A_{\nu}\right)_{\nu \in \mathrm{N}}$ be an increasing sequence of closed disks in $X$. Let $A=\operatorname{span}\left(\bigcup_{v_{N}} A_{\nu}\right)$. Then we have: If codim $A \leqq \boldsymbol{\aleph}_{0}$, then $A$ is closed.

Proof of Lemma 3.4. We prove (Lb). (L), (LC) and (LCb) can 
be proved analogously. As for (2), see the proof of Proposition 2 in [21]. The other proofs are straightforward. Case (Lb): Let $\left(A_{\nu}\right)_{\nu \in N}$ be a (bs) in $A$ and $h$ a seminorm on $A$ such that $h \mid A_{\nu}$ is continuous for every $\nu \in N$. Let $h_{1}$ be a seminorm-continuation of $h$ to $X$. By Theorem 3.4 of [18], there exists for every $\nu \in N$ a continuous semi-norm $k_{\nu}$ on $X$ such that $\left|\left(h_{1}-k_{\nu}\right)(a)\right|<\nu^{-1}$ for all $a \in A_{\nu}$. This shows that $\left(k_{\nu}\right)_{\nu \in N}$ is a Cauchy sequence with respect to the topology of uniform convergence on the sets $A_{\nu}, \nu \in N$, and thus also on the sets $\bar{A}_{\nu}$. Since, by assumption, $\left(\bar{A}_{\nu}\right)_{\nu \in N}$ is a (bs) in $X$ and $X$ has property (Lb), Corollary 3.6 of [18] yields that the sequence $\left(k_{\nu}\right)_{\nu \in N}$ converges to a continuous seminorm $k$ on $X$ with respect to the topology of uniform convergence on the sets $\bar{A}_{\nu}, \nu \in N$. It is easy to see that $k \mid A=h$.

Proof of Proposition 3.5 (2). We prove the case codim $A=\boldsymbol{\aleph}_{0}$. Let $\left(x_{\nu}\right)_{\nu \in N}$ be a Hamel base of a complement of $A$ and let $\left(A_{\nu}\right)_{\nu \in N}$ be a (bs) in $A$. Denote by $\eta$ the finest locally convex topology on $X$ agreeing with $\tau$ on the sets $\bar{A}_{\nu}, \nu \in N$. By Theorem 2.5, the sequence $\left(\bar{A}_{\nu}+\Gamma\left\{x_{1}, \cdots, x_{\nu-1}\right\}\right)_{\nu \in N}$ is a (bs) in $X$; by Lemma 2.6 we have: $\eta\left|\left(\bar{A}_{\nu}+\Gamma\left\{x_{1}, \cdots, x_{\nu-1}\right\}\right)=\tau\right|\left(\bar{A}_{\nu}+\Gamma\left\{x_{1}, \cdots, x_{\nu-1}\right\}\right)$, hence, by assumption, $\eta$ is compatible with the dual pair $\left(X, X^{\prime}\right)$. Theorem 2.4 (1) yields: $\bar{A}=\bigcup_{\nu \in N} \nu \bar{A}_{\nu}$. So $\left(\bar{A}_{\nu}\right)_{\nu \in N}$ is an (as) of closed sets in $\bar{A}$. Since $\left(\bar{A}_{\nu}+\Gamma\left\{x_{1}, \cdots, x_{\nu-1}\right\}\right)_{\nu \in N}$ is a (bs) in $X$ and, by Lemma 1.1, every compact disk is absorbed by an (as) of closed sets, we conclude that $\left(\bar{A}_{\nu}\right)_{\nu \in \mathrm{N}}$ itself is a (bs) in $\bar{A}$.

Other applications of Lemma 3.4 are the following:

1. If $A$ is a linear subspace of an l.c.s. $(X, \tau)$ such that every bounded subset of $X$ is contained in the closure of a bounded subset of $A$, then the assumptions of Lemma 3.4 are fulfilled. This yields an extension of Proposition 6.2, Ch. 1 of [21].

2. Webb ([29], Theorems 9 and 10) proves that under the additional hypothesis that $X^{\prime}=X^{+}$, the space of all sequentially continuous linear functionals on $X$, the conclusion of Theorem 3.1 (2) is valid for subspaces $A$ such that $\operatorname{codim} \bar{A} \leqq \boldsymbol{\aleph}_{0}$ in cases "evaluable" and " $\aleph_{0}$-evaluable". The condition $X^{\prime}=X^{+}$can be replaced by a weaker one:

LEMMA 3.6. Let $(X, \tau)$ be an l.c.s. and let $\mathscr{B}$ be any family of bounded disks in $X$ such that $\bigcap_{B \in \gg} B=X$. Let $A$ be a linear subspace of $X$ and let $\left(A_{\nu}\right)_{\nu \in N}$ be an (as) in $A$ such that

(i) $\operatorname{codim} A$ in $\operatorname{span}\{A \cup B\}$ is finite for every $B \in \mathscr{B}$.

(ii) every $B \cap A, B \in \mathscr{B}$, is absorbed by some $A_{\nu}$.

Denote by $\eta$ the finest locally convex topology on $X$ agreeing with $\tau$ on the sets $\bar{A}_{\nu}, \nu \in N$. Then we have: 
If $X^{\prime}$, endowed with the topology of uniform convergence on the sets $B \in \mathscr{B}$, is complete, then $\eta$ is compatible with the dual pair $\left(X, X^{\prime}\right)$.

Theorem 3.7. Let $(X, \tau)$ be an 1.c.s. such that $\left(X^{\prime}, \beta\left(X^{\prime}, X\right)\right)$ is complete. Let $A$ be a linear subspace of $X$ with property (b) and such that codim $\bar{A} \leqq \boldsymbol{\aleph}_{0}$. Then we have: If $X$ is of type (nb), $n \in\{1, \cdots, 8\} \backslash\{7\}$, or $(\mathrm{Lb})$, then $A$ is of the same type.

The cases $n \in\{3,4\}$ extend Theorems 9 and 10 of [29]. (Observe that there are complete (DF)-spaces such that $X^{\prime} \neq X^{+}$, [28] p. 361.)

Proof of Lemma 3.6. Let $x^{*} \in(X, \eta)^{\prime}$. Then $x^{*} \mid \bar{A}_{\nu}$ is continuous and thus also $x^{*} \mid\left(\bar{A}_{\nu}+\Gamma\left\{x_{1}, \cdots, x_{n}\right\}\right)$ for any $\left\{x_{1}, \cdots, x_{n}\right\} \subset X$. But by Theorem 2.5 every finite union $\bigcup_{1}^{n} B_{i}, B_{i} \in \mathscr{B}$, is absorbed by such a set. Hence, by assumption on $X^{\prime}$ and Grothendieck's completeness theorem, $x^{*} \in X^{\prime}$.

Proof of Theorem 3.7. If $X$ is of type (nb), $n \in\{1, \cdots, 8\} \backslash\{7\}$, or (Lb), then, by Theorem 3.1 (2), $\bar{A}$ is of the same type. Let $\left(A_{\nu}\right)_{\nu \in N}$ be a (bs) in $A$. Combining Theorem 2.4 (1) and Lemma 3.6 we see that $\left(\bar{A}_{2}\right)_{\nu \in N}$ is an (as) in $\bar{A}$. Reasoning as in the proof of Proposition 3.5 (2) we conclude that $\left(\bar{A}_{\nu}\right)_{\nu \in N}$ is a (bs) in $\bar{A}$. Thus $A$ is a linear subspace of $\bar{A}$ which fulfills the assumptions of Lemma 3.4.

\section{A variant of the Banach-Steinhaus theorem.}

Notation. Let $(X, \tau)$ be an l.c.s.

(1) We denote by $C$ its cone of continuous seminorms, by $\sigma_{C}$ the topology of pointwise convergence on $X_{C}=C-C$, and for every $A \subset X$ by $A_{C}^{0}$ the set $A_{C}^{0}=\{h \in C \mid h a \leqq 1$ for every $a \in A\}$ (see [18]).

(2) If $\mathscr{A}=\left(A_{\nu}\right)_{\nu \in N}$ is an (as) in $X$ we say that a filterbase $\mathscr{F}$ on $X^{\prime}$ (resp. on $C$ ) is ultimately $\mathscr{A}$-bounded if for every $\nu \in N$ there exists $F \in \mathscr{F}$ and $\alpha>0$ such that $F \subset \alpha A_{\nu}^{0}$ (resp. $\left.F \subset \alpha\left(A_{\nu}\right)_{C}^{0}\right)$. A net $\left(h_{\lambda}\right)_{\lambda \in A}$ in $X^{\prime}$ (resp. in $C$ ) is said to be ultimately $\mathscr{A}$-bounded if the associated filterbase $\mathscr{F}=\left(F_{\lambda}\right)_{\lambda \in \Lambda}$, where $F_{\lambda}=\left\{h_{\gamma} \mid \gamma \in \Lambda, \lambda \leqq \gamma\right\}$, is ultimately $\mathscr{A}$-bounded. ((2) is motivated by Definition 1 in [2].)

THEOREM 4.1. Let $(X, \tau)$ be an l.c.s. The following assertions are equivalent:

(1) $(X, \tau)$ is barrelled (resp. evaluable).

(2) (i) $(X, \tau)$ carries the Mackey topology.

(ii) For every (as) (resp. (bs)) $\mathscr{A}=\left(A_{\nu}\right)_{\nu \in N}$ in $X$ we have: every 
ultimately $\mathscr{A}$-bounded $\sigma\left(X^{\prime}, X\right)$-Cauchy net in $X^{\prime}$ is $\sigma\left(X^{\prime}, X\right)$-convergent.

(3) For every (as) (resp. (bs)) $\mathscr{A}=\left(A_{\nu}\right)_{\nu \in N}$ in $X$ we have: every ultimately $\mathscr{A}$-bounded $\sigma_{C}$-Cauchy net in $C$ is $\sigma_{C^{-}}$-convergent.

Proof. $\quad(1) \Rightarrow(2)$ : Let $\mathscr{A}=\left(A_{\nu}\right)_{\nu \in N}$ be an (as) (resp. (bs)) in $X$, let $\left(h_{\gamma}\right)_{\gamma \in \Gamma}$ be an ultimately $\mathscr{A}$-bounded $\sigma\left(X^{\prime}, X\right)$-Cauchy net in $X^{\prime}$ and define for $\gamma \in \Gamma F_{\gamma}$ to be the set $F_{\gamma}=\left\{h_{\beta} \mid \beta \in \Gamma, \gamma \leqq \beta\right\}$. Moreover, let $h \in X^{*}$ be the $\sigma\left(X^{*}, X\right)$-limit of $\left(h_{r}\right)_{r \in \Gamma}$. By assumption we choose for every $\nu \in N$ an index $\gamma(\nu) \in \Gamma$ and $\alpha_{\nu}>0$ such that $F_{\gamma(\nu)} \subset$ $\alpha_{\nu} A_{\nu}^{0}$ and $\gamma(\nu) \leqq \gamma(\nu+1), \nu \in N$. Let $\nu \in N$. We have in particular: $F_{\gamma(\nu)} \subset \alpha_{\mu} A_{\mu}^{0}$ for every $\mu \in N, \mu \leqq \nu$. Hence: (i) $F_{\gamma(\nu)} \subset \bigcap_{\mu \leqq \nu}\left(\alpha_{\mu^{-1}} A_{\mu}\right)^{0}=$ $\left(\bigcup_{\mu \leqq \nu} \alpha_{\mu}^{-1} A_{\mu}\right)^{0}=\overline{\left(\Gamma \bigcup_{\mu \leqq \nu} \alpha_{\mu}^{-1} A_{\mu}\right)^{0}}$. Let $B_{\nu}=\overline{\Gamma \bigcup_{\mu \leqq \nu} \alpha_{\mu}^{-1} A_{\mu}}$. Then $\left(B_{\nu}\right)_{\nu \in N}$ is an (as) (resp. (bs)) of closed sets in $X$. By (i) we have: (ii) $F_{r(\nu)}^{*} \subset B_{\nu}^{*}$, where $F_{r(\nu)}^{*}$ denotes the closure of $F_{r(\nu)}$ in $X^{*}$ with respect to $\sigma\left(X^{*}, X\right)$ and $B_{\nu}^{*}$ denotes the polar set of $B_{\nu}$ in $X^{*}$. This yields:

(iii) $h \in \bigcap_{r \in \Gamma} F_{r}^{*} \subset \bigcap_{\nu \in N} F_{\gamma(\nu)}^{*} \subset \bigcap_{\nu \in N} B_{\nu}^{*}=\left(\bigcup_{\nu \in N} B_{\nu}\right)^{*}$. Since $X$ is barrelled (resp. evaluable) there is by [5], Corollary 2b (see Remark 1(a) following Theorem 2.4) a neighbourhood $U$ of 0 in $X$ such that $U \subset \bigcup_{\nu \in N} B_{\nu}$. Hence: (iv) $h \in\left(\bigcup_{\nu \in N} B_{\nu}\right)^{*} \subset U^{*}=U^{0}$, i.e. $h \in X^{\prime}$.

The proof $(2) \Rightarrow(1)$ is straightforward.

Taking into account results in [17], the proof of $(1) \Leftrightarrow(3)$ is essentially the same as for the implications (1) $\Leftrightarrow(2)$.

The following is Definition 1 of [2]: Let $(X, \tau)$ be an l.c.s. and $\mathscr{W}$ the filter of $\beta\left(X^{\prime}, X\right)$-neighbourhoods of zero in $X^{\prime}$.

(i) A net $\left(x_{\gamma}^{\prime}\right)_{r \in \Gamma}$ in $X^{\prime}$ is said to be ultimately bounded, if for every $W \in \mathscr{W}$ there is $\alpha \in \boldsymbol{K}$ and $\gamma_{0} \in \Gamma$ such that $x_{r}^{\prime} \in \alpha W$ for every $\gamma \geqq \gamma_{0}$.

(ii) $(X, \tau)$ is said to be an (ab)-space if it is a Mackey space and if every ultimately bounded $\sigma\left(X^{\prime}, X\right)$-Cauchy net in $X^{\prime}$ is $\sigma\left(X^{\prime}, X\right)$-convergent.

For the special case of (DF)-spaces Theorem 4.1 yields the following result of Valdivia ([27] Theorem 1):

Corollary 4.2. An evaluable (DF)-space is an (ab)-space.

5. Examples. In this section there are specified for each $n \in\{2, \cdots, 9\}$ classes of spaces which are of type (n) but not of type $((n-1) b)$ and spaces of type (L) which do not have property $(\mathrm{Sb})$.

5.1. Quasi-Baire but not b-Baire like:

5.1(1) If $(X, \tau)$ is a reflexive (FG)-space ("countably-normed" in 
the sense of [10], see [8]), then $\left(X^{\prime}, \beta\left(X^{\prime}, X\right)\right)$ is quasi-Baire.

5.1(2) The strong dual of a nonnormable metrizable l.c.s. is not $b$-Baire-like.

In particular, if $X=C_{c}^{\infty}(K), K$ a compact subset of $\boldsymbol{R}^{n}$ ([22], p. 94), then $\left(X^{\prime}, \beta\left(X^{\prime}, X\right)\right)$ is a Ptak-, nuclear, regular (LB)- (hence also ultrabornological (DF)-) space which is quasi-Baire but not $b$-Baire-like ("Regular (LB)-space" in the sense of [8].)

(5.1(1) follows from Lemma 1.4 and the fact that $\left(X^{\prime}, \beta\left(X^{\prime}, X\right)\right)$ is barrelled. 5.1(2) is a consequence of Proposition 1.6.)

5.2. Barrelled but not b-quasi-Baire. If the strong dual of a metrizable l.c.s. $X$ is $b$-quasi-Baire, then there exists a continuous norm on $X$. Hence, the strong dual of a distinguished metrizable l.c.s. on which there exists no continuous norm is barrelled but not $b$-quasi-Baire. In particular, if $X=C^{\infty}\left(\boldsymbol{R}^{n}\right)$, then $\left(X^{\prime}, \beta\left(X^{\prime}, X\right)\right)=\mathscr{E}^{\prime}$, the space of distributions with compact support, is a Ptak-, nuclear, regular (LB)- (hence also ultrabornological (DF)-) space which is not $b$-quasi-Baire.

5.3. The strong dual of a nondistinguished metrizable l.c.s. is $\aleph_{0}$-barrelled but not evaluable.

For examples of $\sigma$-barrelled but not $\boldsymbol{\aleph}_{0}$-evaluable l.c.s. we refer to $[21]$.

5.4. (C) but not $\sigma$-evaluable.

5.4(1). If $X$ is a reflexive (F)-space and $\eta$ a locally convex topology on $Y=X^{\prime}$ such that $\sigma\left(X^{\prime}, X\right) \subset \eta \subset \beta\left(X^{\prime}, X\right)$, then $(Y, \eta)$ has property (C).

5.4(2). The weak dual of an infinite-dimensional (F)-space is not $\sigma$-evaluable. $(5.4(1)$ is a consequence of Smulian's theorem, cf. [13], $\S \S 24.1(2)$ and 2(7); 5.4(2) follows from Corollary 2c of [5], see Remark 1(b) following Theorem 2.4.)

5.5. (S) but not (Cb). Let $(X, \tau)$ be a $\sigma\left(X, X^{\prime}\right)$-sequentially complete (F)-space which is not reflexive, and let $\eta$ be a locally convex topology on $X^{\prime}$ such that $\sigma\left(X^{\prime}, X\right) \subset \eta \subset \tau\left(X^{\prime}, X\right)$. Then $\left(X^{\prime}, \eta\right)$ has (S) but not $(\mathrm{Cb})$. So there are even complete Mackey spaces which are of type $(\mathrm{S})$ but not $(\mathrm{Cb})$. As concrete examples let $(X, \tau)=$ $L^{1}(\mu)$ or $(X, \tau)=l_{1}$ with the usual norm topologies.

5.6. (LC) but not $(\mathrm{Sb})$. Let $(X, \tau)$ be a sequentially complete l.c.s. which is not $\sigma\left(X, X^{\prime}\right)$-sequentially complete, and let $\eta$ be a locally convex topology on $X^{\prime}$ such that $\sigma\left(X^{\prime}, X\right) \subset \eta \subset \tau\left(X^{\prime}, X\right)$. Then $\left(X^{\prime}, \eta\right)$ has property (LC) but not $(\mathrm{Sb})$. So there are even 
Mackey spaces with property (LC) (and thus with property (L)) but not $(\mathrm{Sb})$. As concrete examples for $(X, \tau)$ let $(X, \tau)=C[0,1]$ or $c_{0}$.

Proof. 1. Let $\mathscr{A}=\left(A_{\nu}\right)_{\nu \in N}$ be an (as) of closed sets in $\left(X^{\prime}, \eta\right)$ and let $U$ be any $\tau$-neighbourhood of zero. Then, by Lemma 1.1, $U^{0}$ is absorbed by some $A_{\nu}$. Hence, the topology $\sigma_{\curvearrowright}$ of uniform convergence on the sets $A_{\nu}, \nu \in N$, is finer than $\tau$. Thus $\left(X, \sigma_{\omega}\right)$ is complete and, by Theorem 7 of [16], $\left(X^{\prime}, \eta\right)$ has property (LC). 2. That $\left(X^{\prime}, \eta\right)$ does not have property $(\mathrm{Sb})$ follows from Lemma 1.4.

5.7. (B) but not (LCb):

5.7(1). The weak dual of a barrelled l.c.s. has property (B).

5.7(2). The weak dual of a non complete metrizable and barrelled 1.c.s. does not have property (LCb).

As concrete examples let for any two metrizable barrelled l.c.s. $\left(X_{i}, \tau_{i}\right), i \in\{1,2\},(X, \tau)=X_{1} \otimes_{\pi} X_{2}$, the $\pi$-tensor product of the spaces $\left(X_{i}, \tau_{i}\right)$. (See [11], 6 Example 1 and [1], § 2 Example 10.)

(5.7(1) is a consequence of Lemma 1.1. 5.7(2) follows from Grothendieck's completeness theorem.)

Final remarks. In the following let $\mathscr{B}$ always denote a family of bounded disks in an l.c.s. $X$ which is directed upwards by inclusion and whose elements cover $\mathrm{X}$.

Define an (as) $\left(A_{\nu}\right)_{\nu \in N}$ to be $\mathscr{B}$-bornivorous (abbrev. "( $\left.\left.\mathscr{B}-\mathrm{bs}\right) "\right)$ if every $B \in \mathscr{B}$ is absorbed by some $A_{\nu}$. Then there is an obvious common generalization of the spaces of the "barrelled type" and of the "evaluable type" of our classification scheme to spaces of

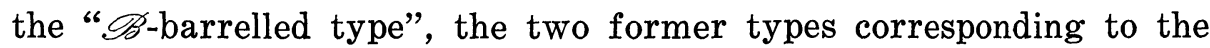
cases of $\mathscr{B}=$ all bounded and finite dimensional disks resp. $\mathscr{B}=$ all bounded disks. Most of the classification- and inheritance-results of this paper can be formulated for the spaces of this " $\mathscr{B}$-barrelled" type.

In particular, if we define an l.c.s. $(X, \tau)$ to be of type (LC $\mathscr{B})$ if for every $(\mathscr{B}$-bs $) \mathscr{A}=\left(A_{\nu}\right)_{\nu \in N}$ we have $\eta_{\mathscr{N}} \subset \tau\left(X, X^{\prime}\right)$, and if a linear subspace $A$ of $X$ is said to have property $(\mathscr{B})$ if $\operatorname{codim}_{\operatorname{span}\{A \cap B\}} A<$ $\infty$ for every $B \in \mathscr{B}$, then the general formulation of Theorem 2.5 yields the corresponding $\mathscr{B}$-versions of Theorem 3.1 and Proposition 3.5. This proposition for example will read then as follows:

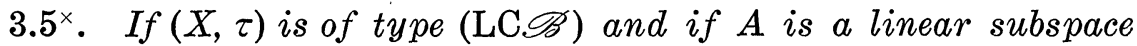
of $X$ of countable codimension and with property $(\mathscr{B})$, then for every $(\mathscr{B} \cap A-\mathrm{bs})\left(A_{\nu}\right)_{\nu \in N}$ in $A$ the sequence $\left(\bar{A}_{\nu}\right)_{\nu \in N}$ is a $(\mathscr{B} \cap \bar{A}-$ bs) in $\bar{A}$. 
This result and the class (LC $\mathscr{B}$ ) seem to be of special interest: after this paper had been completed the author learned of the forthcoming paper [31] of M. Valdivia ${ }^{1}$. On one hand Valdivia treats the finite- (resp. countable-) codimensional inheritance problem for spaces of type (nb) (resp. of type (n)), $n \in\{3,4,5\}$, too. On the other hand, one of the main objects of Valdivia is the inheritance of Mackey-completeness of the dual of an l.c.s. with respect to the topology $\sigma_{\mathscr{B}}$ of uniform convergence on the sets $B \in \mathscr{B}$. Now, concerning this kind of completeness we have the following result:

$\left(X^{\prime}, \sigma_{\infty}\right)$ is Mackey-complete if and only if $(X, \tau)$ has property (LC. $\mathscr{B})$.

By means of this proposition some of the results of [31]-in slightly extended versions- can be deduced from the corresponding $\mathscr{B}$-versions of our results in $\S 3$ : e.g. Proposition $3.5^{\times}$above extends Theorems 1 and 8 of [31]. An extended form of Theorems 5 and 13 of [31] is the following $\mathscr{B}$-version of Theorem 3.1 (1), cases (LC) and $(\mathrm{LCb})$ :

If $A$ is a linear subspace of $(X, \tau)$ of countable codimension and

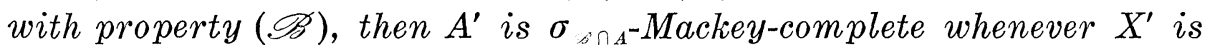
$\sigma_{\mathscr{B}}$-Mackey-complete.

For the sake of completeness we add a proof of the necessity part of Proposition $(*)$ above: Let $\mathscr{A}=\left(A_{\nu}\right)_{\nu \in N}$ be a $(\mathscr{B}-\mathrm{bs})$ in $X$.

(i) For every $B \in \mathscr{B}$ and every $\lambda \in \boldsymbol{R}^{+} \backslash\{0\}$ there exists $\nu(B, \lambda) \in \boldsymbol{N}$ such that $\lambda B \subset \nu A_{\nu}$ for every $\nu \geqq \nu(B, \lambda)$.

Let $x^{*} \in X^{*}$ such that $x^{*} \mid A_{\nu}$ is continuous for every $\nu \in N$. Then, by (i), $x^{*} \in$ completion $_{\sigma_{s}} X^{\prime}$. Moreover: for every $\nu \in N$ there exists $x_{\nu}^{\prime} \in X^{\prime}$ such that $\left|\left(x^{*}-x_{\nu}^{\prime}\right)(a)\right|<\nu^{-3}$ for every $a \in A_{\nu}$ and thus:

(ii) $\left|\nu\left(x^{*}-x_{\nu}^{\prime}\right)(\nu a)\right|<\nu^{-1}$ for every $a \in A_{\nu}$. By (i) and [11, p. 225] we conclude that $\left(x_{\nu}^{\prime}\right)_{\nu \in N}$ is Mackey-convergent to $x^{*}$ in compl ${ }_{\sigma_{\triangle}} X^{\prime}$. Hence, if $\left(X^{\prime}, \sigma_{\mathscr{s}}\right)$ is Mackey-complete, we have $x^{*} \in X^{\prime}$.

\section{REFERENCES}

1. N. Bourbaki, Eléments de Mathématique, Livre V: Espaces Vectoriels Topologiques, Ch. III-V; Paris, Hermann 1955.

2. C. L. DeVito, On Alaoglu's theorem, bornological spaces and the Mackey-Ulam theorem, Math. Ann., 192 (1971), 83-89.

3. M. De Wilde, Vector topologies and linear maps on products of topological vector spaces, Math. Ann., 196 (1972), 117-128.

4. M. De Wilde and C. Houet, On some permanence properties in topological vector spaces, unpublished.

${ }^{1}$ I should like to thank Professor Valdivia for sending me a preprint of this paper. 
5. - On increasing sequences of absolutely convex sets in locally convex spaces, Math. Ann., 192 (1971), 257-261.

6. M. De Wilde, Various types of barrelledness and increasing sequences of balanced and convex sets in locally convex spaces, Proc. Summer School Top. Vector Spaces (Bruxelles, 1972), Lecture Notes Math., 331 (1973), 211-217.

7. D. van Dulst, Barreledness of subspaces of countable codimension and the closed graph theorem, Comp. Math., 24 (1972), 227-234.

8. K. Floret and J. Wloka, Einführung in die Theorie der lokalkonvexen Räume, Lecture Notes in Math. 56, Bln., Hdbg., N. Y., Springer 1968.

9. D. J. H. Garling, A generalized form of inductive-limit topology for vector spaces, Proc. London Math. Soc., (3) 14 (1964), 1-28.

10. I. M. Gelfand and G. E. Silov, Verallgemeinerte Funktionen, Bd. 2, Dtsch. Verl. d. Wiss., Berlin 1963.

11. A. Grothendieck, Espaces vectoriels topologiques, 2. Ed., Sociedade de matemática de Sao Paulo 1958.

12. J. Horvath, Topological Vector Spaces and Distributions, Vol. 1, Reading, Mass., Addison-Wesley, 1966.

13. G. Köthe, Topologische lineare Räume, Bd. 1, Berlin, Hdbg., N. Y., Springer 1966. 14. M. Levin and S. Saxon, A note on the inheritance of properties of locally convex spaces by subspaces of countable codimension, Proc. Amer. Math. Soc., 29 (1971), 97-102. 15. D. A. Raikov, Vollständigkeitskriterien für lokalkonvexe Räume, Uspechi mat. nauk 14, 1 (1959), 223-229 (Russian).

16. W. Roelcke, On the finest locally convex topology agreeing with a given topology on a sequence of absolutely convex sets, Math. Ann. 198 (1972), 57-80.

17. W. Ruess, Ein Dualkegel für p-konvexe topologische lineare Räume, Ber. Ges. Math. Datenv. Bonn, 60 (1972).

18. - A Grothendieck representation for the completion of cones of continuous seminorms, Math. Ann., 208 (1974), 71-90.

19. S. Saxon, Nuclear and product spaces, Baire-like spaces, and the strongest locally convex topology, Math. Ann., 197 (1972), 87-106.

20. S, Saxon and M. Levin, Every countable codimensional subspace of a barrelled space is barrelled, Proc. Amer. Math. Soc., 29 (1971), 91-96.

21. J. Schmets, Espaces associés à un espace linéaire à semi-normes, Publ. Univ. Liège, Analyse fonctionnelle et appl., (1972-73).

22. F. Trèves, Topological Vector Spaces, Distributions and Kernels, Academic Press, N. Y. 1967.

23. M. Valdivia, On (DF)-spaces, Math. Ann., 191 (1973), 38-43.

24. — A hereditary property in locally convex spaces, Ann. Inst. Fourier, 21 (2) (1971), 1-2.

25. — Absolutely convex sets in barrelled spaces, Ann. Inst. Fourier 21 (2) (1971), $3-13$.

26. — On final topologies, J. für Math., 251 (1971), 193-199.

27. - On a class of quasi-barrelled spaces, Math. Ann., 202 (1973), 295-300.

28. J. H. Webb, Sequential convergence in locally convex spaces, Proc. Camb. Phil. Soc., 64 (1968), 341-364.

29. - Countable-codimensional subspaces of locally convex spaces, Proc. Edinburgh Math. Soc., 18 (1973), 167-172.

30. - Subspaces of bornological and quasi-barrelled spaces, J. London, Math. Soc., (2), 7 (1973), 157-158.

31. M. Valdivia, On quasi-completeness and sequential completeness in locally convex spaces, to appear in J. für Math.

Received March 27, 1975 and in revised form December 8, 1975. 


\title{
PACIFIC JOURNAL OF MATHEMATICS
}

\author{
EDITORS
}

\author{
RICHARD ARENS (Managing Editor) \\ University of California \\ Los Angeles, California 90024
}

\author{
R. A. Beaumont \\ University of Washington \\ Seattle, Washington 98105
}

J. DugundjI

Department of Mathematics

University of Southern California

Los Angeles, California 90007

D. Gilbarg and J. Milgram

Stanford University

Stanford, California 94305

\section{ASSOCIATE EDITORS}
E. F. BECKENBACH
B. H. NEUMANN
F. WOLF
K. Yoshida

\section{SUPPORTING INSTITUTIONS}

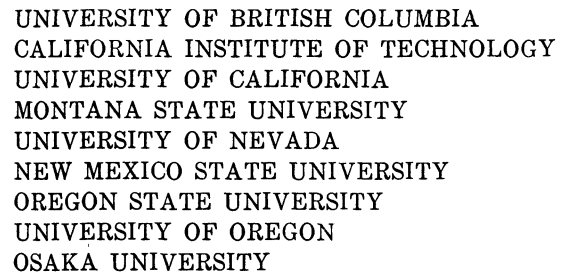

UNIVERSITY OF BRITISH COLUMBIA CALIFORNIA INSTITUTE OF TECHNOLOGY UNIVERSITY OF CALIFORNIA MONTANA STATE UNIVERSITY UNIVERSITY OF NEVADA NEW MEXICO STATE UNIVERSITY OREGON STATE UNIVERSITY UNIVERSITY OF OREGON OSAKA UNIVERSITY

\author{
UNIVERSITY OF SOUTHERN CALIFORNIA \\ STANFORD UNIVERSITY \\ UNIVERSITY OF HAWAII \\ UNIVERSITY OF TOKYO \\ UNIVERSITY OF UTAH \\ WASHINGTON STATE UNIVERSITY \\ UNIVERSITY OF WASHINGTON

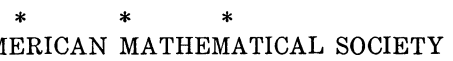

The Supporting Institutions listed above contribute to the cost of publication of this Journal, but they are not owners or publishers and have no responsibility for its content or policies.

Mathematical papers intended for publication in the Pacific Journal of Mathematics should be in typed form or offset-reproduced, (not dittoed), double spaced with large margins. Please do not use built up fractions in the text of your manuscript. You may however, use them in the displayed equations. Underline Greek letters in red, German in green, and script in blue. The first paragraph or two must be capable of being used separately as a synopsis of the entire paper. Items of the bibliography should not be cited there unless absolutely necessary, in which case they must be identified by author and Journal, rather than by item number. Manuscripts, in triplicate, may be sent to any one of the editors. Please classify according to the scheme of Math. Reviews, Index to Vol. 39. All other communications should be addressed to the managing editor, or Elaine Barth, University of California, Los Angeles, California, 90024.

The Pacific Journal of Mathematics expects the author's institution to pay page charges, and reserves the right to delay publication for nonpayment of charges in case of financial emergency.

100 reprints are provided free for each article, only if page charges have been substantially paid. Additional copies may be obtained at cost in multiples of 50 .

The Pacific Journal of Mathematics is issued monthly as of January 1966. Regular subscription rate: $\$ 72.00$ a year (6 Vols., 12 issues). Special rate: $\$ 36.00$ a year to individual members of supporting institutions.

Subscriptions, orders for back numbers, and changes of address should be sent to Pacific Journal of Mathematics, 103 Highland Boulevard, Berkeley, California, 94708.

PUBLISHED BY PACIFIC JOURNAL OF MATHEMATICS, A NON-PROFIT CORPORATION

Printed at Kokusai Bunken Insatsusha (International Academic Printing Co., Ltd.), 8-8, 3-chome, Takadanobaba, Shinjuku-ku, Tokyo 160, Japan.

Copyright (C) 1975 by Pacific Journal of Mathematics Manufactured and first issued in Japan 


\section{Pacific Journal of Mathematics}

\section{Vol. 63, No. 2 \\ April, 1976}

Joseph Anthony Ball and Arthur R. Lubin, On a class of contractive perturbations

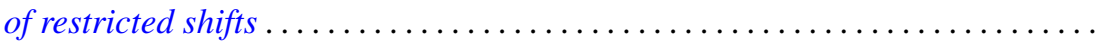

Joseph Becker and William C. Brown, On extending higher derivations generated

by cup products to the integral closure .......................

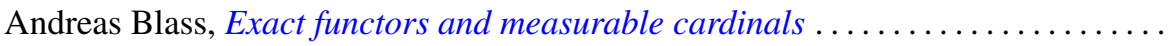

Joseph Eugene Collison, A variance property for arithmetic functions . . . . . . . . . .

Craig McCormack Cordes, Quadratic forms over nonformally real fields with a

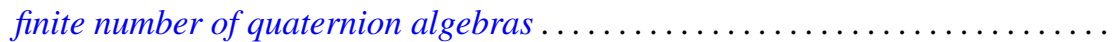

Freddy Delbaen, Weakly compact sets in $H^{1} \ldots \ldots \ldots \ldots \ldots \ldots \ldots \ldots \ldots$

G. D. Dikshit, Absolute Nörlund summability factors for Fourier series ..........

Edward Richard Fadell, Nielsen numbers as a homotopy type invariant. . ........

Josip Globevnik, Analytic extensions of vector-valued functions . . . . . . . . . . . .

Robert Gold, Genera in normal extensions . . . . . . . . . . . . . . . . . . . 389

Solomon Wolf Golomb, Formulas for the next prime

Robert L. Griess, Jr., The splitting of extensions of $S L(3,3)$ by the vector space

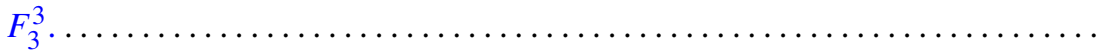

Thomas Alan Keagy, Matrix transformations and absolute summability .........

Kazuo Kishi, Analytic maps of the open unit disk onto a Gleason part.

Kwangil Koh, Jiang Luh and Mohan S. Putcha, On the associativity and commutativity of algebras over commutative rings . ..... . .

James C. Lillo, Asymptotic behavior of solutions of retarded differential difference equations.

John Alan MacBain, Local and global bifurcation from normal eigenvalues ..

Anna Maria Mantero, Sets of uniqueness and multiplicity for $L^{p}$

J. F. McClendon, Embedding metric families

L. Robbiano and Giuseppe Valla, Primary powers of a prime ideal .

Wolfgang Ruess, Generalized inductive limit topologies and barrelledness

properties.

Judith D. Sally, Bounds for numbers of generators of Cohen-Macaulay ideals

Helga Schirmer, Mappings of polyhedra with prescribed fixed points and fixed point indices.

Cho Wei Sit, Quotients of complete multipartite graphs

S. Sznajder and Zbigniew Zielezny, Solvability of convolution equations in $\mathscr{K}_{p}^{\prime}$,

$p>1$.

Mitchell Herbert Taibleson, The existence of natural field structures for finite

dimensional vector spaces over local fields

William Yslas Vélez, A characterization of completely regular fields

P. S. Venkatesan, On right unipotent semigroups ..............

Kenneth S. Williams, A rational octic reciprocity law ............

Robert Ross Wilson, Lattice orderings on the real field .......... 